\title{
Harveian Oration 2020: Elucidation of molecular oxygen sensing mechanisms in human cells: implications for medicine
}

\author{
Author: Peter ] Ratcliffe $\mathrm{A}^{\mathrm{A}}$
}

KEYWORDS: oxygen sensing, oxygen homeostasis, HIF hydroxylase system

DOI: 10.7861/clinmed.ed.22.1.harv

\section{Introduction}

I am honoured to present tonight's Harveian Lecture, albeit in the slightly unusual circumstances mandated by COVID-19. It will be a personal story, but I hope one which will bring some encouragement to intending physician scientists considering a programme of work in discovery science, as I was as a young nephrologist a little over 30 years ago.

I will describe my laboratory's work on the molecular dissection of cellular oxygen-sensing mechanisms and the pathways that they control. The subject has a clear connection with Harvey's revolutionary work on the function of the heart and the circulation of the blood, since the carriage of oxygen to the tissues is one of the main functions of the blood, and that is where I'll begin.

Apart from giving an overview of the findings themselves and their role in physiological oxygen homeostasis, I'd like to highlight the twists and turns of the experimental work, in particular the way that knowledge builds on knowledge in unexpected ways. Abraham Flexner, the US educationist, wrote a famous essay in the early twentieth century on the 'usefulness of useless knowledge'.' I'd like to paraphrase Flexner by emphasising the 'importance of incomplete knowledge'. Our politicians are impatient for the utility of knowledge, perhaps not unreasonably, and our editors, perhaps less reasonably, are so often concerned with its completeness. Of course, most in tonight's audience will know that neither qualification of knowledge is valid. But nevertheless, I wish to emphasise the concerns I have, in case, in the face of societal or government impatience, or even that of our own peers, we lose our way.

Author: ${ }^{\text {A }}$ rofessor of clinical medicine, Ludwig Institute for Cancer Research, University of Oxford, Oxford, UK, and director of clinical research, Francis Crick Institute, London, UK

\section{Oxygen homeostasis: the historical background}

Harvey's work demonstrated the function of the heart as a pump, the nature of the arterial pulse, and the importance of valves in heart and veins in ensuring the unidirectional flow of blood. He inferred the circulation of the blood, including, in higher animals with a biventricular heart, its serial passage from the right heart through the lungs to the left heart and hence to the systemic circulation. Aside from the sheer extent and revolutionary status of these discoveries, the work is remarkable for the total absence of a dependence on new technology. All of the deductions were based on simple visual observations, 'ocular experiments' as they are described his major work Exercitatio Anatomica de Motu Cordis et Sanguinis in Animalibus (1628). The work revolutionised thinking: the first correct conclusions about the function of an internal organ, derived by careful observations, which could have been, but were not, performed centuries earlier. Yet the work on the circulation of the blood was clearly incomplete in that Harvey's work provided no obvious insight into its function. This troubled some of his detractors, but not especially Harvey, who wrote:

insomuch as to those who do still reject the Circulation because they see neither the efficient nor the final cause of it, there remains, because I have as yet joined nothing to it, only to say this much: First you must confess that there is a Circulation, before you enquire for what it is.

It was just as well Harvey did not concern himself too much with the 'for what it is', since more than a century would pass before advances in chemistry made that a feasible goal. Though Newton's Principia (1687) fuelled ambition for scientific insights into life, by and large the subsequent 'age of reason' was a disappointment in biological and medical terms.

One clue that Harvey could have added, but apparently didn't, was provided later in the seventeenth century by Richard Lower, an Oxford physician working with Robert Hooke, who noted the change in colour as the blood passed through the lungs and largely deduced the function of the lungs, writing: 'nitrous spirit of the air, vital to life is mixed with the blood as it passes through the lungs'. The nature of that 'nitrous spirit of the air' (again incomplete knowledge) had to wait for the discovery of oxygen by Scheele, Priestley and Lavoisier. Although the priority and independence among these three in the discovery of oxygen is 
debated, there is no doubt who provided the critical chemical insights. Using a quantitative approach, including the careful measurement of changes in weight, Antione Lavoisier correctly identified the chemical nature of combustion and demonstrated its stoichiometric equivalence with biological metabolism. For instance, using quantitative methods he demonstrated that heat generated by combustion (of charcoal) and by metabolism (of a guinea pig) was associated with production of the same quantities of 'fixed air' (carbon dioxide).

Unfortunately, Lavoisier's productivity came to an untimely end. His activities in the Ferme Generale, a much-hated institution that collected taxes for the French state (at a profit), put him on the wrong side of the French revolutionary authorities. His young wife Marie-Ann Paulze Lavoisier, who had married him aged 13, appealed for clemency on grounds of his eminence and utility as a scientist, but her pleas were in vain. The response of the president of the Revolutionary Court, Jean-Baptiste Coffinhal, 'La Republique n'a pas besoin de savants ni de chimistes,' was swiftly followed by Lavoisier's execution by the guillotine on 8 May 1794. Who knows how much was lost. The government of the First Republic later saw it as a mistake and apologised to his widow; their impatience was surely a source of unspeakable grief for Madame Lavoisier, but, one might hope, a lesson to others in government.

Arguably, Lavoisier's demonstration of the chemical stoichiometric equivalence of combustion and metabolism was the first success in reductionist biology; evidence that life after all could be explained by chemistry. But there was a major difference: oxidative metabolism is subject to extremely tight levels of control, whereas combustion is uncontrolled and potentially explosive. Of the many characteristics of life, this is perhaps the most fundamental; the essential quality of the chemistry of life is the control that ultimately underpins physiological homeostasis and the complexities of replication.

Another French scientist, Claude Bernard, did much to promote this thinking. Though the term itself, homeostasis, was introduced later by the US physiologist Walter B Cannon, Bernard first developed the concept, viewing disease as a disturbance of homeostasis. His particular focus was on the mechanisms by which higher animals defend their milieu interiore, a constant internal environment that enables them to live independently of the conditions of their external environment. Water volumes, metabolic stores, temperature and oxygen concentration were identified as targets of such controls.
However, it was Bernard's protégé (his 'preparateur' at the College de France and later successor as professor of physiology at the Sorbonne) Paul Bert who developed the work on oxygen, showing that it was low oxygen (hypoxia) that was responsible for symptoms (and death) at high altitude, work that is set out in detail in his 1877 magnum opus La pression barometrique. ${ }^{2}$

At Bert's suggestion, another French investigator, Francois Gilbert Viault, made careful measurements of blood cells at sea level and on the Andean plateau, clearly establishing the adaptive response of erythropoiesis to high altitude. ${ }^{3}$ It was the extreme sensitivity of this response that was the inspiration and starting place for the work I'm going to describe. This was most clearly demonstrated by the work of Mabel FitzGerald, an unsung female scientist who was a member of the Oxford-Yale expedition to Pike's Peak, Colorado in the summer of $1911 .{ }^{4}$ While the remainder of the party (all male) made measurements of altitude adaption over time in a convenient hotel at the summit, FitzGerald travelled on her own to the surrounding mining towns to make measurements in fully adapted individuals living at intermediate altitudes. The story is that this rather asymmetric division of labour was on account of her gender and the absence of a chaperone at the summit hotel. Whether true or not, FitzGerald's data (Fig 1) provided clarity on the sensitivity of the erythropoietic response to altitude and hence hypoxia. It was a direct derivation of this work, the extraordinary sensitivity of the erythropoietin production by the kidneys and liver to hypoxic stimuli, that drew me as a young nephrologist to the field in the late 1980s. I felt that the mechanism of sensing was likely to be important and might just be tractable.

\section{Our research journey: the quest for cellular oxygen-sensing mechanisms}

Here I'd like to pause and reflect for moment. For this (the selection of the research question) is the most important consideration for success as a scientist. As may become evident in this lecture, it is much more important than profound intelligence or extreme skill as an investigator. But the selection of a research question is not straightforward and not helped by the current environment facing a new investigator. I have argued that the history of research on oxygen homeostasis encompasses the first piece of human physiology (Harvey), the first piece of reductionist biology (Lavoisier), one of the first considerations in homeostasis itself, a necessary condition for life (Bernard). All were
Fig 1. Mabel FitzGerald, pioneering female scientist who accompanied J.S. Haldane's expedition to study adaptation to altitude on Pike's Peak, Colorado. a) FitzGerald measuring haemoglobin concentration in the blood, by dilution and colour matching to a standard (photograph from the Herald Telegraph, Colorado Springs, 8 July 1911). b) FitzGerald's measurements of haemoglobin in adapted subjects residing at different altitudes. The results demonstrate the effect of even small differences in altitude reflecting the high sensitivity to hypoxia.

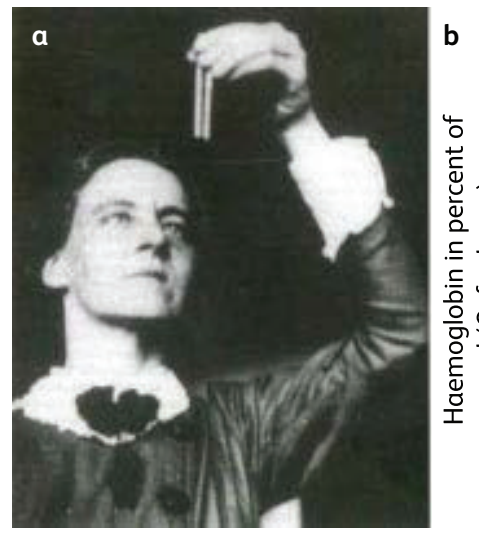

\section{b}

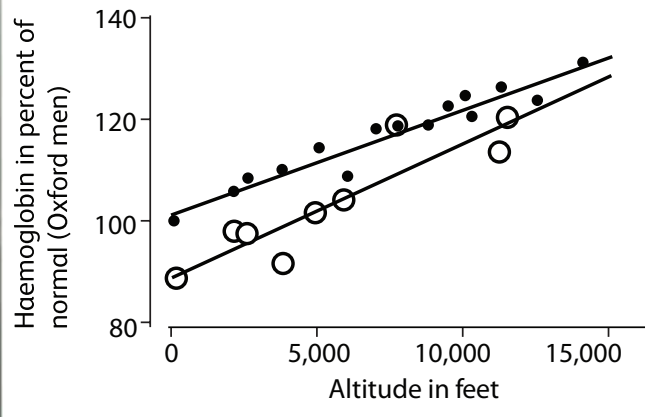


established centuries ago. So, the first consideration is to ask why the molecular basis of oxygen homeostasis was largely passed over by the great and good of twentieth century science. I'd like to consider two factors.

The first factor was that attempts on the problem were indeed made, but the experimental systems (largely intact animals) were too complex. This is a key consideration; attempts to draw conclusions from interventions on highly heterogeneous systems, though sometimes all that is possible, will always be difficult. Claude Bernard himself wrote extensively on the importance (and difficulty) of integrating experimental findings made in different systems to understand physiology. In the case of oxygen homeostasis, the number of different interactions of oxygen with biological processes make this a particular challenge. Thus, at the time I came to be interested in the field, significant efforts had made to understand mechanisms of oxygen sensing but had not yielded clear insights.

Next, I suspect largely as a result of this, came the second factor: the field simply wasn't very fashionable. This is important to understand. Scientists are just as susceptible to fashion as other human beings (ie massively so). At the present time, this susceptibility is unhelpfully reinforced by excessive peer review and the excessive power of the commercial scientific press. So, apart from strictly scientific considerations, one problem facing the young investigator is how to deal with fashion. In considering the research options facing today's new investigators, versus those available to William Harvey, it is often said that the 'low hanging fruit' have been taken: that depletion of the pool of soluble scientific problems has made their task harder. I do not think this is correct. The problem is not that scientific advances have depleted the opportunities for discovery; almost certainly they have greatly expanded them. The problem is that these dazzling advances and the charismatic leaders who made them are distracting. They are influential on appointments committees, publish in 'high profile' places and attract young investigators into fields that are already crowded or solved or both. For myself, the first problem (that of an appropriate experimental system) was solved by H Franklin Bunn, a haematologist at Harvard University, who demonstrated the hypoxia-inducible production of erythropoietin in two liver (hepatoma) derived tissue culture cell lines, thus providing a much simpler experimental system. ${ }^{5}$ By a series of miracles I avoided the second trap, and was very fortunate to become generously funded to study my chosen question as a Wellcome Trust senior clinical fellow, without a track record of publication in the field.

Apart from its extraordinary sensitivity to hypoxia, a second characteristic of erythropoietin regulation had suggested that underlying this response was a specific mechanism for the sensing of oxygen, as opposed to some secondary metabolic compromise associated with hypoxia. Observations in the early twentieth century had identified excessive erythrocytosis as a manifestation of chronic cobalt poisoning. ${ }^{6}$ Subsequent work showed that cobalt, at erythropoietic doses, is not a metabolic poison and that metabolic inhibitors such as cyanide do not have this action., ${ }^{7,8}$ Thus, it was concluded that the response of the erythropoietin gene to hypoxia reflected the function of a specific and sensitive 'oxygen sensor'. It was generally believed that this sensing system would be a specific property of specialised cells within the kidneys and liver that make erythropoietin; hence, a substantial part of our initial effort was directed into attempts to identify and isolate these cells from kidneys. The work failed in respect of generating a tissue culture model from kidney, which we needed for molecular analyses. For reasons which are still unclear, erythropoietin gene expression is strongly suppressed by most forms of kidney injury and I suspect similar processes underlie its suppression in explanted kidney cells. However, the work did clearly identify the cells, a population of renal interstitial fibroblasts which somewhat surprisingly had no obviously specialised features. ${ }^{9}$

Franklin Bunn's hepatoma cells were therefore critical for opening the field to molecular analysis, providing clear evidence that regulation of erythropoietin production by oxygen was a cell autonomous function. Our initial aim was to identify the oxygendependent DNA regulatory sequences that we assumed must lie at the erythropoietin locus and then follow the interactions of these sequences upstream through what we considered might be a complex signal transduction cascade to the postulated oxygen sensor. Though, reassuringly, the hepatoma cells responded to cobalt as in intact animals, not everyone believed that these hepatoma cells harboured the most important physiological 'oxygen sensor', since they were derived from liver not kidney. Nevertheless, we (Christopher Pugh and I) enthusiastically embarked on the first step: transfection studies of sequences at the erythropoietin locus that identified a powerful oxygenregulated control sequence, a transcriptional enhancer, lying 3' to the mouse erythropoietin gene. ${ }^{10}$ These sequences are highly conserved and lie at exactly the same position with respect to the erythropoietin gene as human sequences identified in parallel work by both Gregg Semenza and Jaime Caro. ${ }^{11,12}$

\section{A widespread system of oxygen sensing in human and animal cells}

We recognised that identification of this oxygen-sensitive transcriptional enhancer would allow the question of cell specificity to be addressed. Was it really true that oxygen sensing was a property that was specific to erythropoietin-producing cells? How would these sequences behave if introduced into cells that did not produce erythropoietin? Initial experiments gave an unclear result: at least the sequences did not appear to be as active as in the erythropoietin-producing hepatoma cells. This initially reinforced the idea of specific erythropoietin-producing cells and I set about what I thought was an innovative (though in retrospective very naive) experiment, attempting to 'expression clone' components of the 'oxygen sensing' pathway by transfer of genes from the 'oxygen sensing' hepatoma cells, to a line that would be predicted to be oxygen-insensitive (ie every cell type, according to the then-existing prejudice). To my surprise, when setting up controls for these experiments, I observed clear oxygen-regulated activity in the cells selected as recipients. Of course, this disqualified the approach, but fundamentally changed our thinking.

We found that responses to hypoxia depended on the culture conditions, but that, when rapidly dividing relatively dense cultures were used, essentially all the cultured cells we tested supported oxygen-regulated activity of the erythropoietin enhancer. Another trainee nephrologist, Patrick Maxwell, did much of this work. Almost certainly, the dependence of responses on tissue culture conditions that Patrick identified related to the dependence of the actual oxygen concentration at the cell monolayer on the oxygen consumption rate of the cells, and hence on their growth rate and density. We were excited by the implications of these findings; the same oxygen-sensing system was clearly widespread, at least 
in mammalian cells, and there must be other targets in nonerythropoietin-producing cells.

The findings, however, met with some scepticism and initial difficulty in publication. From my current vantage point, I have some sympathy with the editor. Part of the concern was again about incomplete knowledge. It was indeed (as was pointed out in review) only one new fact, and we hadn't then followed through by addressing the obvious next question: what were the additional oxygen-sensitive targets that were implied? I had little experience of research practice and it didn't occur to me that deriving followon conclusions, or a 'more complete story', might be important before publication. As far as I was concerned the findings were robust and the implications clear. The findings were eventually published, but not in the journal of first intent. ${ }^{13}$

Our findings were soon corroborated by data from Gregg Semenza, who, importantly, had identified a transcription factor in hepatoma cells that bound the erythropoietin control sequences in an oxygen-dependent manner, which he termed hypoxia-induced factor (HIF). ${ }^{14}$ In keeping with our transfection results, HIF was found to be expressed widely across different cell types. ${ }^{15}$ So what were its targets? The efforts in my laboratory initially focused on genes encoding enzymes in the glycolytic pathway, glucose transporters and angiogenic growth factors, whose regulation we considered might contribute to oxygen homeostasis. With the help of other clinical trainees, including Ben Ebert, John Firth and Jonathan Gleadle, who joined the laboratory and contributed much to the work, we were rapidly able to show that many of these genes demonstrated the characteristics that had been defined for erythropoietin (induction by both hypoxia and cobaltous ions) and possessed oxygen-regulated control sequences, with the same core DNA sequence that was present in the erythropoietin enhancer and bound to HIF. ${ }^{16-18}$ In other work that proved important later on in the story, we went on to show that insect (Drosophila melanogaster) cell extracts also possessed a hypoxiainducible activity that bound these sequences. ${ }^{19}$ Thus, the system was conserved in species that do not have an erythropoietin gene or even possess an oxygen-carrying blood vascular system similar to that in higher animals.

At the time we were rather surprised that so many genes, involved in different processes and manifesting very different regulatory characteristics in the intact organism, were apparently responsive to the identical transcriptional system. But as technical innovations enhanced the capacity to assay gene expression, we were truly astonished by the unexpected breadth of the HIF transcriptional cascade (Fig 2). ${ }^{20}$ Many different genes with roles in processes such as $\mathrm{pH}$ regulation, matrix metabolism, iron transport and cell differentiation were revealed to be controlled by HIF. Studies using gene arrays and 'next-generation sequencing' identified hundreds or thousands of HIF transcriptional targets. $^{21,22}$ Many of these transcripts encoded transcript factors themselves; others encompassed different classes of regulatory RNA. ${ }^{23,24}$ Together, these regulatory cascades might be predicted to impinge on practically every aspect of cell physiology, as might have been (but was not) predicted from the central role of oxygen in biological chemistry.

Other methods, particularly analyses of the phenotypes associated with genetic inactivation of components of the HIF system in the tissues and organs of recombinant mice, revealed entirely unexpected insights into the role of HIF in different aspects of integrated animal physiology. Studies by Randall Johnson and others revealed that genetic inactivation of HIF in immune and inflammatory cells is associated with phenotypes that imply important functions in these cells. ${ }^{25-27}$ Coupled with evidence for physiological hypoxia in regions of the lymph node, bone marrow

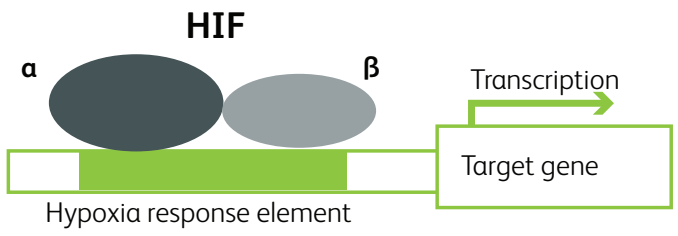

Signalling through hypoxia inducible factor

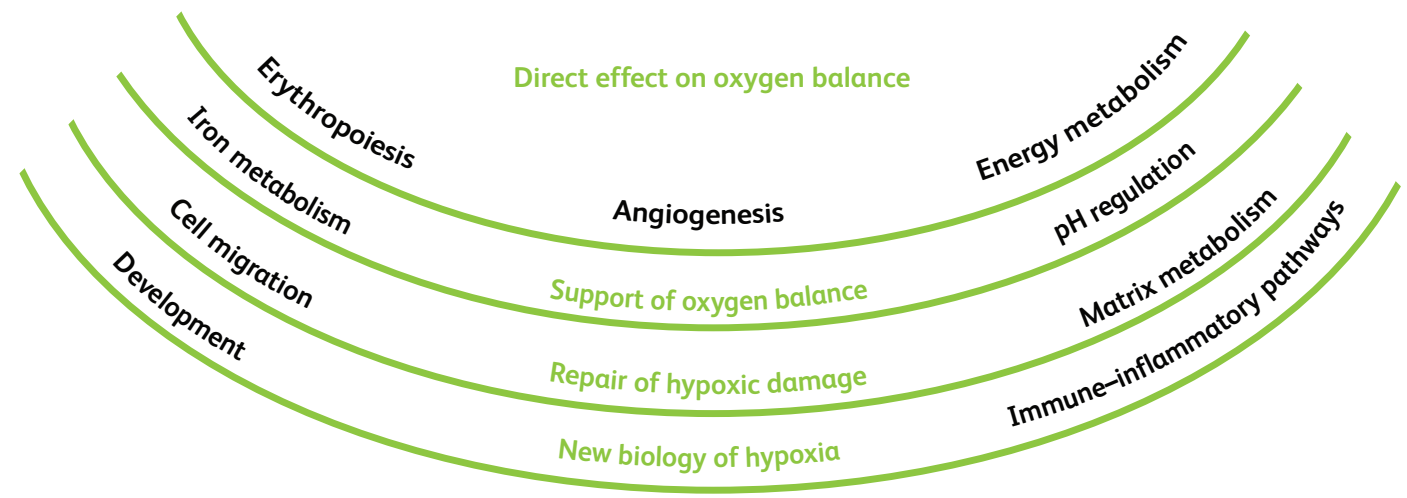

Fig 2. Schematic illustrating the transcriptional response transduced by HIF (hypoxia inducible factor). HIF binds as an $\alpha / \beta$ heterodimer to hypoxia responses elements that are associated with HIF transcriptional target genes. HIF target genes mediate a very broad array of cellular and systemic responses to hypoxia, which reveal that adaptation to hypoxia extends more widely than was previously foreseen. 
and thymus, and the pathological generation of profound hypoxia at sites of inflammation and immune activation, the work suggests a new physiology of hypoxia in immune and inflammatory control. One interesting possibility is that hypoxia (via HIF) has a direct effect on regulatory T cell function. ${ }^{28}$ However, linking of the effects of ablative genetic interventions to a clearly defined regulatory role in physiology is not straightforward and much of the rapidly expanding knowledge on the HIF transcriptional cascade remains to be fully understood at the level of integrated physiology. Nevertheless, the work on erythropoietin, once considered to represent a tightly restricted response to hypoxia, clearly opened completely unexpected new fields of research. One might ask how it was that so much was overlooked? I am not sure, but our tendency to overlook things that have been overlooked by others is remarkable, something that must surely have surprised William Harvey himself.

\section{Molecular mechanism of the oxygen sensing}

I'll return to the unexpected reach of the HIF transcriptional cascade later, in the context of therapeutics. At the time our main focus was to dissect pathways that lay upstream of HIF, connecting its regulation to the availability of oxygen. Gregg Semenza had purified HIF and identified the encoding cDNAs, demonstrating that the DNA binding complex was an alpha/ beta heterodimer of basic helix-loop-helix PAS (Per-AHR-ARNTSim) transcription factors (Fig 2). ${ }^{29}$ The beta subunit (HIF-1 $\beta$ ) had previously been identified as a dimerisation partner of the aryl hydrocarbon receptor, whereas the alpha subunit (HIF-1 $\alpha$ ) was a novel protein that is specifically involved in the response to hypoxia.

So, what lay upstream of HIF-1 $\alpha$ ? We had no idea how far we had to travel, nor exactly what we were looking for. Based on the mimicry of the response to hypoxia by iron chelators and cobaltous ions, it was widely believed that some form of ferroprotein (with which these substances might interact) was the source of the oxygen-sensitive signal. But almost every type of ferroprotein had been considered, with the exception of those that we were later to show actually function in this way. Presuming the existence of a long and complex transduction pathway connecting this 'sensor' to gene transcription and not being 100\% confident I possessed the necessary skills for a direct biochemical approach to its analysis, we set up almost every conceivable alternative (genetic and other) strategy to dissect the pathway in parallel. In the end it was a mixture of several of these approaches that proved successful. I'm not going to describe all the work; some was embarrassingly naive on my part and some heroic on the part of others. Morwenna Wood and Emma Vaux, two more trainee nephrologists who joined the laboratory, approached a world record in tissue culture in their isolation of somatic mutant cells that were defective in signalling hypoxia. Both ultimately succeeded in generating mutants in the pathway, ${ }^{30,31}$ although the means to identify the defective genes in mammalian cells were at the time insufficiently rapid for our purpose.

I'll outline some of the steps in the path we ultimately followed, which I hope illustrate my theme of how knowledge builds on knowledge in unexpected ways. Our first direct approach to the problem was the identification of sequences in HIF-1 $\alpha$ (and later the paralogue HIF-2 $\alpha$ ) that would confer oxygen sensitivity when fused to a heterologous protein. We found three sequences that had this property; two operated by conferring oxygen-dependent protein instability on the fusion protein and one appeared to operate independently of any change in protein stability. ${ }^{32,33}$ This 'domain architecture' was conserved between the paralogues HIF- $1 \alpha$ and HIF- $2 \alpha$ and we looked for a common consensus sequence, confidently expecting to find the postulated protein phosphorylation motif that would lead us to the upstream signalling pathway. Our efforts went as far as to mutate every phospho-acceptor in one of these domains, but the oxygensensitive property was unaltered. ${ }^{32}$ The work was again difficult to publish on the basis of being 'incomplete knowledge' and of course largely negative data. But in fact, at least for us, it was directional in excluding the 'mainstream' hypothesis regarding signal transduction.

The next key step was the recognition that the von Hippel-Lindau (VHL) protein, a commonly mutated tumour suppressor protein in kidney cancer, interacted with two of these domains (those that operated through protein stability). This work interfaces with contributions from my other co-laureate in Stockholm, Bill Kaelin. Bill and others had found that certain hypoxia-inducible transcripts were constitutively upregulated in VHL-defective cells, but the mechanism was unclear. ${ }^{34,35}$ Using antibodies raised 'in house' to both the paralogues, HIF- $1 \alpha$ and HIF- $2 \alpha$, we made the connection to HIF, revealing that VHL physically interacted with the previously defined 'oxygen-dependent degradation' domains in HIF- $\alpha$ polypeptides. ${ }^{36,37}$ We showed that VHL was necessary for oxygen-dependent degradation of HIF- $\alpha$ polypeptides, acting as the recognition component of a conditional ubiquitin E3 ligase that targets HIF- $\alpha$ polypeptides for proteasomal destruction in the presence of oxygen. ${ }^{36,37}$

In turn, this work framed the question as to what governed the association between HIF- $\alpha$ polypeptides and the VHL protein. We found that recombinant HIF- $\alpha$ proteins or synthetic peptides needed exposure to a cell extract in the presence of oxygen, before they could physically associate with $\mathrm{VHL}$, indicating that an oxygen-dependent modification of HIF- $\alpha$ was essential. Our analyses of this process ultimately revealed that the key modification was the hydroxylation of specific prolyl residues in the HIF- $\alpha$ polypeptide. The work involved a combination of biochemical characterisation of the HIF-modify activity, genetic mutational approaches, mass spectrometry, and candidacy. Fig 3 depicts the 'Eureka moment' when predictions based on this work were confirmed; a synthetic peptide bearing a trans4-hydroxyprolyl residue rather than proline at position 564 bound to VHL constitutively, without the need for any treatment with cell extract. These findings, and independent work from Bill Kaelin's laboratory, immediately suggested a mechanism of oxygen sensing. ${ }^{38,39}$ The then known prolyl hydroxylases that catalyse procollagen prolyl hydroxylation to stabilise the collagen triple helix are 2-oxoglutarate-dependent dioxygenases. As dioxygenases (enzymes that split dioxygen and incorporate both oxygen atoms into their substrates) they have an absolute requirement for molecular oxygen. In the catalytic cycle, the oxidation of the prime substrate (in this case proposed to be the HIF peptide containing the target prolyl residue) is coupled to the oxidative decarboxylation of the Krebs cycle intermediate 2-oxoglutarate, yielding succinate and carbon dioxide. ${ }^{40}$ In keeping with established properties of erythropoietin and HIF, these enzymes are also classically inhibited by cobaltous ions and iron chelators. The procollagen prolyl hydroxylases themselves had not seemed likely candidates, as the target prolyl peptide in HIF- $\alpha$ bore no resemblance to the typical PPG repeats that are targeted 


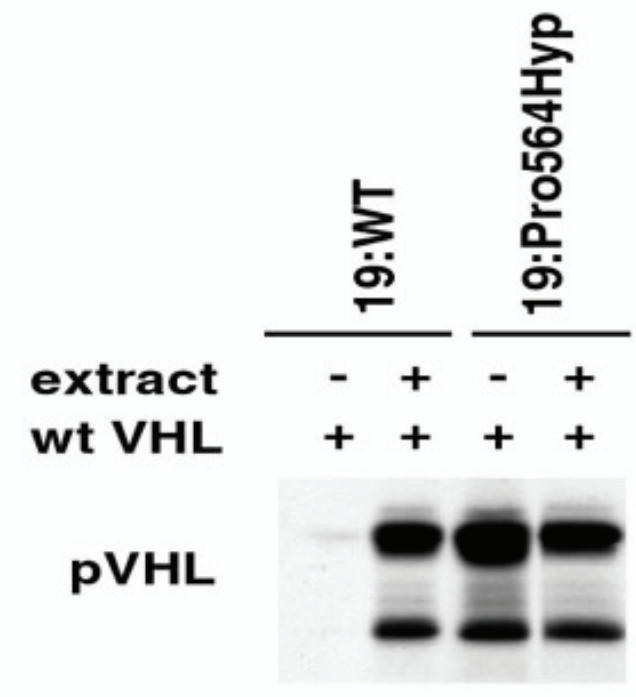

Fig 3. Definition of prolyl hydroxylation as the regulatory modification of HIF-a proteins governing binding to the von HippelLindau protein (VHL). Binding of VHL to synthetic peptides derived from human HIF-1a sequences (556-574) was assayed by pull-down. The unmodified HIF peptide (19:WT) requires treatment with cell extract to promote binding of VHL. In contrast a HIF peptide bearing a trans-4hydroxyprolyl substitution at residue 564 (19:Pro564Hyp) binds VHL without such treatment.

by the procollagen prolyl hydroxylases. In collaborative work, Johanna Myllyharju from Oulu, Finland demonstrated directly that procollagen prolyl hydroxylase had no activity on the HIF peptide. So we were clearly looking for another type of human prolyl hydroxylase, with the properties of a 2-oxoglutarate-dependent dioxygenase, which we knew from the earlier work must be conserved between human and invertebrate animals.

Looking back this might well have been recognised, and driven thinking, decades earlier by linking the classical (and highly distinctive) finding of induction of erythropoiesis by cobalt poisoning ${ }^{41}$ and the action of ascorbate to antagonise this effect (reported in the 1930s) ${ }^{42}$ to the properties of procollagen prolyl hydroxylases and related enzymes, which were identified as ascorbate-requiring enzymes that are inhibited by cobalt many years earlier. ${ }^{43-45}$ But that is in retrospect; the work now directed the search for one or more 2-oxoglutarte dependent dioxygenases that catalyse HIF prolyl hydroxylation to generate the oxygendependent signal controlling VHL-dependent degradation.

To our good fortune, one of the leading authorities on this family of enzymes, Christopher Schofield, was also working in Oxford, in the Department of Chemistry. Chris's input was key in identifying the enzymes and he became a long-term collaborator in this and much subsequent work. The story of how scientific knowledge derived from apparently unconnected lines of work may come to together to answer an unanticipated question is one I'd like to emphasise. Chris had worked on 2-oxoglutarate dependent dioxygenases and related enzymes that are produced by microorganisms and involved in antibiotic synthesis. ${ }^{46}$ But the $X$-ray structures he obtained on these enzymes also provided the basis for structurally informed predictions of many other putative 2-oxoglutarate dependent dioxygenases encoded by the human genome. Some of these, like the HIF system, were highly conserved across invertebrate animals. In an entirely different line of work, researchers working on the nematode worm Caenorrhabditis elegans had been painstakingly cataloguing mutants with different types of developmental abnormality. One piece of work by Bob Horvitz and colleagues identified 145 mutants with a 'egg-laying defective' phenotype due to neuromuscular defects that lead to an inability to extrude their eggs. ${ }^{47}$ Following our earlier attempts to trace the phylogeny of the HIF system in Drosophila cells, a post-doc in the laboratory, John O'Rourke, identified a putative orthologue of HIF- $\alpha$ in C elegans. Critically, he raised an antibody to this protein to enable us to test worms bearing mutations in genes that were hypothesised to be involved in the oxygen-sensing process. The antibody worked beautifully; simply exposing worms to an atmosphere of $1 \%$ oxygen in a bell jar massively induced HIF- $\alpha$, as assayed by immunoblotting. But none of the mutant worms we had tested had shown any alteration in this response.

However, very interestingly one of the putative human 2-oxoglutarate-dependent dioxygenases that Chris Schofield had indicated was a good candidate, and which was highly conserved in $C$ elegans, corresponded to one of the mutants catalogued as 'egg-laying defective' by Bob Horvitz, egl-9. The mutants were duly ordered from the very well curated $C$ elegans repository. Another of those Eureka moments followed shortly, with Andy Epstein, a trainee cardiologist and PhD student who had taken on the $C$ elegans work, bursting through the office door with the result (Fig 4). In contrast with wild-type worms (and the multiple other mutants we had tested), all worms bearing mutant alleles in egl-9 showed constitutive upregulation of the HIF- $\alpha$ protein, irrespective of oxygen levels, as would be expected if their 'oxygen sensing' enzyme was defective. ${ }^{48}$ We were rapidly able to confirm that egl9 did indeed encode a HIF prolyl hydroxylase and that in humans there are three paralogues bearing the highly conserved catalytic domain, which we called PHD (prolyl hydroxylase domain) 1, 2 and $3 .^{49}$ In human HIF- $1 \alpha$ and HIF- $2 \alpha$ there are two sites of prolyl hydroxylation, each of which can independently promote VHLdependent proteolysis, accounting for two of three domains in HIF- $\alpha$ polypeptides that had been shown to independently confer oxygen-regulated activity on heterologous proteins earlier in the work. The third oxygen-regulated domain was later shown to be subjected to hydroxylation by a second type of 2-oxoglutaratedependent dioxygenase that catalyses the hydroxylation of

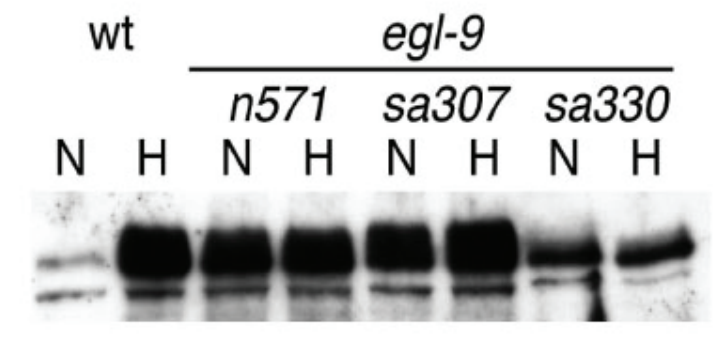

Fig 4. Identification of the 'oxygen sensing' HIF prolyl hydroxylase in the nematode worm $C$ elegans. ${ }^{48}$ Immunoblot of $C$ elegans HIF- $\alpha$ protein using an antibody produced 'in house' to test responses of wildtype and mutant worms to hypoxia. Whereas HIF- $\alpha$ is strongly induced by hypoxia in wild-type worms (wt), HIF- $\alpha$ protein is stabilised and upregulated irrespective of oxygen in worms bearing different mutant alleles of the gene encoding the HIF proyl hydrolylase egl-9. 


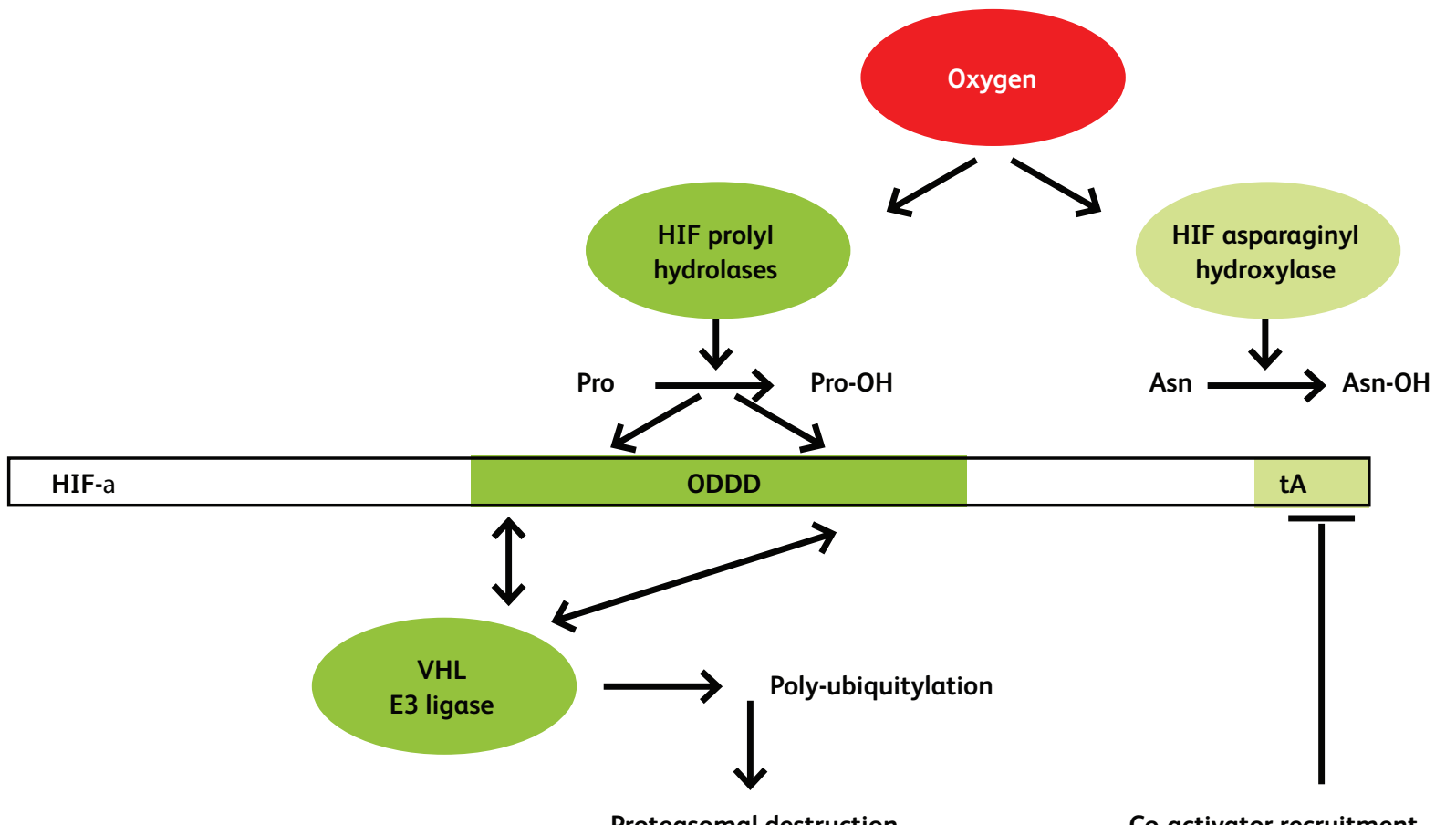

Fig 5. Schematic illustrating the regulation of HIF by prolyl and asparaginyl hydroxylation. HIF prolyl hydroxylases target two sites in the oxygendependent degradation domain (ODDD) of HIF- $\alpha$ sub-units to promote association with the von Hippel-Lindau (VHL) ubiquitin E3 ligase and destruction by the ubiquitin proteasome pathway. A HIF asparaginyl hydroxylase targets the C-terminal transcriptional activation domain (tA) to prevent association with co-activators. In hypoxia, hydroxylation of HIF- $\alpha$ is suppressed allowing it to escape proteolytic destruction and form an active transcriptional complex.

specific asparaginyl residue to reduce transcriptional activity, at least in part by blocking the recruitment of transcriptional coactivators to the HIF complex. ${ }^{49-51}$ Thus, HIF is subject to a dual mode of regulation, being both degraded and inactivated in the presence of oxygen (Fig 5).

With a few possible exceptions, all animals possess at least one representative of each of the key components of the HIF-PHDVHL system and most also possess a HIF asparaginyl hydroxylase. Gene duplication events at the base of vertebrate evolution generated additional copies of several components. ${ }^{52}$ Thus, humans possess three isoforms of HIF- $\alpha$, and three isoforms of the HIF prolyl hydroxylase. Given the interest in selective therapeutic manipulation of HIF pathway in human disease, an interesting question raised by these findings concerns the extent that these duplicated isoforms are specifically involved in the development or regulation of the complex oxygen delivery systems such as the heart, lungs and blood vessels that are found in higher animals and which are often the target of medical intervention. Some evidence for this is seen in the functions of the paralogues HIF- $1 \alpha$ and HIF- $2 \alpha$. Two of the classical adaptations to hypoxia, increased erythropoiesis and increased ventilatory sensitivity to hypoxia (characteristics of altitude acclimatisation so beautifully defined in FitzGerald's early studies), appear to be specifically dependent on HIF- $2 \alpha,{ }^{53,54}$ whereas a number of genes involved in the more conserved metabolic functions, such as glycolysis, appear to be specifically dependent on HIF- $1 \alpha .{ }^{55} \mathrm{HIF}-2 \alpha$ manifests much greater tissue specificity of expression than HIF-1 $\alpha$ and in keeping with the above roles, HIF- $2 \alpha$ is very highly expressed in the interstitial fibroblasts of the kidney ${ }^{56}$ (which synthesise erythropoietin) and in the chemo-sensitive cells of the carotid body (that are important in the hypoxic control of ventilation). ${ }^{57,58}$ Nevertheless, across the genome, in different cells, HIF-1 $\alpha$ and HIF- $2 \alpha$ each have a very extensive and partially overlapping set of transcriptional targets that extend well beyond these functions. ${ }^{59}$ So even isoform-specific therapeutic manipulation of HIF would need to take account of pleiotropic biological actions.

\section{Oxygen sensing in different kingdoms of life}

As already outlined, we were initially surprised that the HIF hydroxylase system operated widely across human cells and was not confined to the regulation of erythropoietin. Given the central importance of oxygen homeostasis to most forms of life, we were next surprised that it appeared restricted to the animal kingdom. Interestingly, subsequent work has now revealed all four eukaryotic kingdoms in fact use enzymatic protein oxidation, but of different types, coupled to proteostasis to signal oxygen levels. Some of these systems appear closely related to the HIF hydroxylase pathway. Thus, the slime-mould Dictyostelium discoideum uses a similar oxygen sensitive prolyl-4-hydroxylase that targets a ubiquitin ligase directly, as opposed its substrates. ${ }^{60}$ Fission yeast deploy a different type of prolyl hydroxylase, a prolyl3-hydroxylase, to regulate highly oxygen-dependent biosynthetic pathways such a sterol synthesis. ${ }^{61}$ Although this system has a biochemical orthologue in human cells, ${ }^{62}$ we have so far been unable to define the physiological function, the oxygen-dependent regulation of cholesterol synthesis in human cells being connected to the HIF pathway. ${ }^{63}$ One system which particularly interested us, 
as it apparently bears no molecular similarities to the HIF system, yet uses the same principle, is that which operates in plants to control the proteolytic stability of ethylene response factor (ERF)VII transcription factors. ${ }^{64,65}$ Here, the enzymatic oxidation is of a different type, the dioxygenation of $\mathrm{N}$-terminal cysteine residues, revealed on putative substrates by methionine aminopeptidases. $\mathrm{N}$-cysteine dioxygenation in plants is catalysed by a series of enzymes known as the plant cysteine oxidases; ${ }^{66}$ following this oxidation, substrates are targeted for degradation by the $\mathrm{N}$-degron pathway. ${ }^{67}$ Thus, plants and animals use conceptually similar (yet somewhat counterintuitive) system of synthesis and oxygen-dependant degradation to signal oxygen levels. As part of examining mechanisms contributing to oxygen homeostasis in human cells more completely, we found that human cells encode an enzyme (ADO, 2-aminoethanethiol dioxygenase, otherwise known as cysteamine dioxygenase) that is similar to the plant cysteine oxidases and catalyses $\mathrm{N}$-cysteine dioxygenation of a series of human proteins. ${ }^{68}$ Among its targets, this system controls the stability of a set of G-protein regulators, RGS (regulator of G-protein signalling) 4, 5 and 16; direct proteolytic regulation of these signalling molecules potentially contributing to oxygen homeostasis on a shorter time-scale than transcriptional pathways mediated by HIF.

\section{The therapeutic implications of the work}

In the final part of the lecture I'd like to return to the HIF system and briefly consider the therapeutic implications. The work I've described completed the intended journey, from erythropoietin to oxygen. But there were surprises at every stage. A massive and wholly unforeseen transcriptional cascade that extends well beyond erythropoietin and erythropoiesis. No complex protein phosphorylation cascade. A direct connection to oxygen involving a then unprecedented mode of signalling through enzymatic protein oxidation coupled to protein degradation. Although, as a clinician, I was aware of the importance of hypoxia in most human disease, it would misleading to claim that this and the associated therapeutic possibilities were the inspiration for the work. Nevertheless, it is rather satisfying that there are now two classes of drug licensed for clinical use that target the HIF hydroxylase system.

One class of drug exploits the use of 2-oxoglutarate as cosubstrate by the HIF hydroxylases, in a strategy that aligns with the classical concept of a drug targeting a discrete site of interaction with a small molecule. Analogues of 2-oxoglutarate that bind the catalytic iron centre of these enzymes but do not support catalysis have the potential to slow the rate of HIF hydroxylation and hence mimic hypoxia. Inhibition of the PHD enzymes alone (ie without inhibition of the HIF asparaginyl hydroxylase) is sufficient to induce much of the HIF response. Relatively specific inhibitors of the PHD enzymes have been developed that efficiently activate HIF and are effective in the treatment of anaemia associated with kidney diseases. Remarkably, with appropriate dose adjustment, such agents appear able to increase erythropoietin production and haemoglobin levels with little evidence of non-erythropoietic systemic actions that might have been predicted from the multiple targets of the HIF transcriptional cascade. ${ }^{69,70}$

The second class of agent aims to inhibit HIF and is nonclassical in that it attempts to target the transcription factor directly. Though most transcription factors have been considered 'undruggable', compounds have been developed that bind a pocket in the PAS domain of HIF- $2 \alpha$ to selectively block dimerisation of HIF-2 and inhibit its transcriptional activity. ${ }^{71}$ These agents are showing great promise in the treatment of VHL-defective renal carcinoma and hereditary VHL disease. ${ }^{72}$ Pharmaceutical interventions therefore have the potential to upregulate or downregulate aspects of the physiological response to hypoxia. Their development aligns with thinking introduced by Claude Bernard, himself a physician. Bernard argued that disease may be viewed as a perturbation of homeostasis and by implication that treatment should targeted at the dysregulated physiological pathways as closely as possible. In some ways targeting of the HIF system is consistent with this and in some ways it isn't. For instance, the physiological stimulation of erythropoiesis by the hypoxic induction of erythropoietin production is supported by HIF through processes such as changes in iron metabolism and direct actions on the marrow. So in the treatment of anaemia it might possible to improve on monotherapy with large doses of erythropoietin. Furthermore, a large number of preclinical studies support the potential of PHD inhibitors to ameliorate ischaemic injury. ${ }^{73}$ On the other hand, the enormous reach of the HIF transcriptional cascade across biology creates a challenge for the ideal intervention: one that would specifically target the components of that cascade that are desired in a particular medical context, but not other components.

Similar considerations apply to the HIF-2 antagonists. The targeting of HIF- 2 in clear cell renal carcinoma and $\mathrm{VHL}$-disease follows evidence that growth of these tumours appears to be driven by the upregulation and constitutive activation of HIF-2, in part through activation of vascular endothelial growth factor (VEGF) signalling. The possibility of targeting upstream HIF-2 pathways, instead or in addition to VEGF, is appealing. But again, the complexity of the HIF system, even that of HIF-2 alone, across both neoplastic and non-neoplastic tissues has the potential to generate other effects, both desired and undesired. . $^{7,75}$

So, although the clinical launch of these new classes of drugs is rightly a source of great optimism, there are uncertainties. Importantly, however, their availability will allow direct clinical observation that may refine or perhaps extend treatment indications. As we enter this new phase of the work, there will be an element of risk, sadly borne by our patients.

\section{Conclusion}

I'll finish by asking the question: what might we (I mean discovery scientists) do better? To what extent have we completed a molecular understanding of oxygen homeostasis that might be securely applied to medicine? We have certainly defined a biochemical reaction that measures at least one type of "error signal' for oxygen availability, an essential component of homeostasis. But how precise responses are shaped across the thousands of HIF transcription targets, how these responses interact across the human body's $\sim 40$ trillion cells, how different physiological oxygen gradients ranging more than an order of magnitude across healthy tissues are accommodated, how these pathways interact with hundreds of other signalling processes, how the need to maintain oxygen homeostasis across widely different time scales is achieved, are still largely unknown. Perhaps we have explained a billionth part of oxygen homeostasis. In 
preparing this lecture I was struck by Haldane's admission in his address to the Harveian Society of New York in 1916, shortly after the studies of altitude adaptation at Pike's Peak:

He who looks for definite 'causal chains' in physiological phenomena finds in place of them a network of apparently infinite complexity. The physiologists who led the revolt of last century against vitalism did not see this network. ${ }^{76}$

I don't think that any longer we would be tempted by the concept of vitalism, but the complexity of biological circuitry created by Darwinian evolution is indeed staggering. What is also misunderstood, or at least underestimated, by those seeking rapid and predictable progress is the way evolution (and hence the biological basis of medicine) runs counter to rational design. Processes are used over and over again and ultimately for quite dissimilar tasks. Thus, it turned out (unexpectedly) that HIF is used to regulate both erythropoiesis and glycolysis. It turned out (unexpectedly) that prolyl hydroxylation is used to generate stability in the collagen triple helix and the oxygen sensitive signals that control HIF. It turned out (unexpectedly) that energetically costly circuits of enzymatic oxidation, breakdown and re-synthesis of transcription factors are deployed to signal oxygen levels across different kingdoms of life. Perhaps a reflection of primitive processes that remove oxidised molecules? Who knows, but none of this helps rational prediction, either in biology or medicine.

As Francois Jacob so elegantly put it, 'Nature is a tinkerer, not an engineer'. What he might have added was that the intelligent and ambitious human mind generally aspires to be an engineer, not a tinkerer. This causes difficulties. Science will deliver medicines, but it is important to be patient and accept a level of uncertainty.

\section{Acknowledgement}

I am grateful to all those who contributed to, and supported, the work in my laboratory though their involvement in its science, funding and administration, particularly to Christopher Pugh and Patrick Maxwell, who were centrally involved in much of the work I have described.

\section{References}

1 Flexner A. The usefulness of useless knowledge. Harper's Magazine 1939;179:544-52.

2 Bert P. La pression barometrique. Paris: Masson, 1878

3 Viault F. Sur l'augmentation considerable du nombre des globules rouges dans le sang chez les habitants des hauts plateaux de l'Amerique du Sud. Comptes Rendu Academie Science Paris 1890;111:917-8.

4 FitzGerald MP. The changes in the breathing and the blood at various high altitudes. Philos Trans $R$ Soc Lond B Biol Sci 1913;203:351-71.

5 Goldberg MA, Glass GA, Cunningham JM, Bunn HF. The regulated expression of erythropoietin by two human hepatoma cell lines. Proc Natl Acad Sci U S A 1987;84:7972-6.

6 Waltner K, Waltner K. Kobalt und blut. Klinische Wochenschrift 1929;8:313.

7 Warren CO, Schubmehl QD, Wood IR. Studies on the mechanism of cobalt polycythemia. Am J Physiol 1944;142:173-8.

8 Necas E, Thorling EB. Unresponsiveness of erythropoietin-producing cells to cyanide. Am J Physiol 1972;222:1187-90.

9 Maxwell PH, Osmond MK, Pugh CW et al. Identification of the renal erythropoietin-producing cells using transgenic mice. Kidney Int 1993:44:1149-62.
10 Pugh CW, Tan CC, Jones RW, Ratcliffe PJ. Functional analysis of an oxygen-regulated transcriptional enhancer lying 3' to the mouse erythropoietin gene. Proc Natl Acad Sci U S A 1991;88:10553-7.

11 Beck I, Ramirez S, Weinmann R, Caro J. Enhancer element at the 3 '-flanking region controls transcriptional response to hypoxia in the human erythropoietin gene. J Biol Chem 1991;266:15563-6.

12 Semenza GL, Nejfelt MK, Chi SM, Antonarakis SE. Hypoxiainducible nuclear factors bind to an enhancer element located 3 ' to the human erythropoietin gene. Proc Natl Acad Sci U S A 1991;88:5680-4.

13 Maxwell PH, Pugh CW, Ratcliffe PJ. Inducible operation of the erythropoietin 3' enhancer in multiple cell lines: evidence for a widespread oxygen sensing mechanism. Proc Natl Acad Sci U S A 1993:90:2423-7.

14 Semenza GL, Wang GL. A nuclear factor induced by hypoxia via de novo protein synthesis binds to the human erythropoietin gene enhancer at a site required for transcriptional activation. Mol Cell Biol 1992;12:5447-54.

15 Wang GL, Semenza GL. General involvement of hypoxia-inducible factor 1 in transcriptional response to hypoxia. Proc Natl Acad Sci U S A 1993:90:4304-8.

16 Firth JD, Ebert BL, Pugh CW, Ratcliffe PJ. Oxygen-regulated control elements in the phosphoglycerate kinase 1 and lactate dehydrogenase A genes: similarities with the erythropoeitin 3' enhancer. Proc Natl Acad Sci U S A 1994;91:6496-500.

17 Gleadle JM, Ebert BL, Firth JD, Ratcliffe PJ. Regulation of angiogenic growth factor expression by hypoxia, transition metals, and chelating agents. Am J Physiol 1995;268:C1362-8.

18 Ebert BL, Firth JD, Ratcliffe PJ. Hypoxia and mitochondrial inhibitors regulate expression of glucose transporter-1 via distinct cis-acting sequences. J Biol Chem 1995;270:29083-9.

19 Nagao M, Ebert BL, Ratcliffe PJ, Pugh CW. Drosophila melanogaster SL2 cells contain a hypoxically inducible DNA binding complex which recognises mammalian HIF-1 binding sites. FEBS Letters 1996:387:161-6.

20 Mole DR, Blancher C, Copley RR et al. Genome-wide association of hypoxia-inducible factor (HIF)-1alpha and HIF-2alpha DNA binding with expression profiling of hypoxia-inducible transcripts. J Biol Chem 2009:284:16767-75.

21 Schödel J, Oikonomopoulos S, Ragoussis ] et al. High-resolution genome-wide mapping of HIF-binding sites by ChIP-seq. Blood 2012:117:e207-17.

22 Xia X, Lemieux ME, Li W, Carroll JS et al. Integrative analysis of HIF binding and transactivation reveals its role in maintaining histone methylation homeostasis. Proc Natl Acad Sci U S A 2009;106:4260-5.

23 Kulshreshtha R, Ferracin M, Wojcik SE et al. A microRNA signature of hypoxia. Mol Cell Biol 2007;27:1859-67.

24 Choudhry H, Schödel J, Oikonomopoulos S et al. Extensive regulation of the non-coding transcriptome by hypoxia: role of HIF in releasing paused RNApol2. EMBO Rep 2014;15:70-6.

25 Cramer T, Yamanishi Y, Clausen BE et al. HIF-1 $\alpha$ is essential for myeloid cell-mediated inflammation. Cell 2003;112:645-57.

26 Walmsley SR, Print C, Farahi N et al. Hypoxia-induced neutrophil survival is mediated by HIF- $1 \alpha$-dependent NF-KB activity. J Exp Med 2005;201:105-15.

27 Dang EV, Barbi J, Yang HY et al. Control of T(H)17/T(reg) balance by hypoxia-inducible factor 1. Cell 2011;146:772-84.

28 Yamamoto A, Hester J, Macklin PS et al. Systemic silencing of PHD2 causes reversible immune regulatory dysfunction. $]$ Clin Investig 2019;129:3640-56.

29 Wang GL, Jiang B-H, Rue EA, Semenza GL. Hypoxia-inducible factor 1 is a basic-helix-loop-helix-PAS heterodimer regulated by cellular $\mathrm{O}_{2}$ tension. Proc Natl Acad Sci U S A 1995;92:5510-14.

30 Wood SM, Wiesener MS, Yeates KM et al. Selection and analysis of a mutant cell line defective in the hypoxia-inducible factor-1 
$\alpha$-subunit (HIF-1 $\alpha$ ): characterisation of HIF-1 $\alpha$ dependent and independent gene expression. J Biol Chem 1998;273:8360-8.

31 Vaux EC, Wood SM, Cockman ME et al. Selection of mutant CHO cells with constitutive activation of the HIF system and inactivation of the von Hippel-Lindau tumor suppressor. J Biol Chem 2001:276:44323-30

32 Pugh CW, O'Rourke JF, Nagao M, Gleadle JM, Ratcliffe PJ. Activation of hypoxia inducible factor-1; definition of regulatory domains within the $\alpha$ subunit. J Biol Chem 1997;272:11205-14.

33 O'Rourke JF, Tian Y-M, Ratcliffe PJ, Pugh CW. Oxygen-regulated and transactivating domains in endothelial PAS protein 1: comparison with hypoxia inducible factor-1 $\alpha$. J Biol Chem 1999;274:2060-71.

34 Iliopoulos O, Levy AP, Jiang C, Kaelin WG, Goldberg MA. Negative regulation of hypoxia-inducible genes by the von Hippel-Lindau protein. Proc Natl Acad Sci U S A 1996;93:10595-9.

35 Gnarra JR, Zhou S, Merrill MJ et al. Post-transcriptional regulation of vascular endothelial growth factor mRNA by the product of the VHL tumor suppressor gene. Proc Natl Acad Sci U S A 1996:93:10589-94.

36 Maxwell PH, Wiesener MS, Chang G-W et al. The tumour suppressor protein $\mathrm{VHL}$ targets hypoxia-inducible factors for oxygendependent proteolysis. Nature 1999;399:271-5.

37 Cockman ME, Masson N, Mole DR et al. Hypoxia inducible factor- $\alpha$ binding and ubiquitylation by the von Hippel-Lindau tumor suppressor protein. J Biol Chem 2000;275:25733-41.

38 Jaakkola P, Mole DR, Tian Y-M et al.Targeting of HIF- $\alpha$ to the von Hippel-Lindau ubiquitylation complex by $\mathrm{O}_{2}$-regulated prolyl hydroxylation. Science 2001;292:468-72.

39 Ivan $\mathrm{M}$, Kondo $\mathrm{K}$, Yang $\mathrm{H}$ et al. HIF $\alpha$ targeted for VHL-mediated destruction by proline hydroxylation: implications for $\mathrm{O}_{2}$ sensing. Science 2001;292:464-8.

40 Schofield C], Ratcliffe PJ. Oxygen sensing by HIF hydroxylases. Nat Rev Mol Cell Biol 2004;5:343-54.

41 Berk L, Burchenal JH, Castle WB. Erythropoietic effect of cobalt in patients with or without anemia. N Engl J Med1949;240:754-61.

42 Barron AG, Barron ESG. Mechanism of cobalt polycythemia. Effect of ascorbic acid. Proc Soc Exp Biol Med 1936;35:407-9.

43 Hutton J], Trappel AL, Udenfriend S. Requirements for alphaketoglutarate, ferrous ion and ascorbate by collagen proline hydroxylase. Biochem Biophys Res Commun 1966;24:179-84.

44 Anttinen H, Ryhanen L, Oikarinen A. Effects of divalent cations on collagen biosynthesis in isolated chick embryo tendon cells. Biochim Biophys Acta 1980;609:321-8.

45 Perry D, Abraham EP, Baldwin JE. Factors affecting the isopenicillin N synthetase reaction. Biochem J 1988;255:345-51.

46 Roach PL, Clifton IJ, Fulop V et al. Crystal-structure of isopenicillin $\mathrm{N}$-synthase is the first from a new structural family of enzymes. Nature 1995:375:700-4

47 Trent C, Tsung N, Horvitz HR. Egg-laying defective mutants of the nematode Caenorhabditis elegans. Genetics 1983;104:619-47.

48 Epstein ACR, Gleadle JM, McNeill LA et al. C. elegans EGL-9 and mammalian homologues define a family of dioxygenases that regulate HIF by prolyl hydroxylation. Cell 2001;107:43-54.

49 Lando D, Peet DJ, Whelan DA, Gorman J], Whitelaw ML. Asparagine hydroxylation of the HIF transactivation domain: a hypoxic switch. Science 2002;295:858-61.

50 Lando D, Peet DJ, Gorman J] et al. FIH-1 is an asparaginyl hydroxylase enzyme that regulates the transcriptional activity of hypoxiainducible factor. Genes Dev 2002;16:1466-71.

51 Hewitson KS, McNeill LA, Riordan MV et al. Hypoxia inducible factor (HIF) asparagine hydroxylase is identical to Factor Inhibiting HIF (FIH) and is related to the cupin structural family. J Biol Chem 2002;277:26351-5.

52 Loenarz C, Coleman ML, Boleininger A et al. The hypoxia-inducible transcription factor pathway regulates oxygen sensing in the simplest animal, Trichoplax adhaerens. EMBO Rep 2011;12:63-70.
53 Paliege A, Rosenberger C, Bondke A et al. Hypoxia-inducible factor2alpha-expressing interstitial fibroblasts are the only renal cells that express erythropoietin under hypoxia-inducible factor stabilization. Kidney Int 2010;77:312-8.

54 Hodson EJ, Nicholls LG, Turner PJ et al. Regulation of ventilatory sensitivity and carotid body proliferation in hypoxia by the PHD2/ HIF-2 pathway. J Physiol 2016;594:1179-95.

$55 \mathrm{Hu}$ C], Iyer S, Sataur A et al. Differential regulation of the transcrip tional activities of hypoxia-inducible factor 1 alpha (HIF-1 $\alpha$ ) and HIF-2 $\alpha$ in stem cells. Mol Cell Biol 2006;26:3514-26.

56 Rosenberger C, Mandriota SJ, Jurgensen JS et al. Expression of hypoxia-inducible factor- $1 \alpha$ and $-2 \alpha$ in hypoxic and ischemic rat kidneys. J Am Soc Nephrol 2002;13:1721-32.

57 Zhou T, Chien M-S, Kaleem S, Matsunami H. Single cell transcriptome analysis of mouse carotid body glomus cells. J Physiol 2016:594:4225-51.

58 Fielding JW, Hodson EJ, Cheng X et al. PHD2 inactivation in Type I cells drives HIF-2alpha-dependent multilineage hyperplasia and the formation of paraganglioma-like carotid bodies. J Physiol 2018;596:4393-412.

59 Smythies JA, Sun M, Masson N et al. Inherent DNA-binding specificities of the HIF-1alpha and HIF-2alpha transcription factors in chromatin. EMBO Rep 2019;20:e46401.

60 van der Wel H, Ercan A, West CM. The Skp1 prolyl hydroxylase from Dictyostelium Is related to the hypoxia-inducible factor- $\alpha$ class of animal prolyl 4-hydroxylases. J Biol Chem 2005;280: $14645-55$.

61 Hughes BT, Espenshade PJ. Oxygen-regulated degradation of fission yeast SREBP by Ofd1, a prolyl hydroxylase family member. EMBO ] 2008;27:1491-501.

62 Singleton RS, Liu-Yi P, Formenti F et al. OGFOD1 catalyzes prolyl hydroxylation of RPS 23 and is involved in translation control and stress granule formation. Proc Natl Acad Sci U S A 2014;111:4031-6.

63 Hwang S, Nguyen AD, Jo Y et al. Hypoxia-inducible factor 1alpha activates insulin-induced gene 2 (Insig-2) transcription for degradation of 3-hydroxy-3-methylglutaryl (HMG)-CoA reductase in the liver. J Biol Chem 2017;292:9382-93.

64 Licausi F, Kosmacz M, Weits DA et al. Oxygen sensing in plants is mediated by an $\mathrm{N}$-end rule pathway for protein destabilization. Nature 2011:479:419-22.

65 Gibbs DJ, Lee SC, Isa N et al. Homeostatic response to hypoxia is regulated by the $\mathrm{N}$-end rule pathway in plants. Nature 2011;479:415-8.

66 Weits DA, Giuntoli B, Kosmacz M et al. Plant cysteine oxidases control the oxygen-dependent branch of the $\mathrm{N}$-end-rule pathway. Nat Commun 2014:5:1-10.

67 Varshavsky A. N-degron and C-degron pathways of protein degradation. Proc Natl Acad Sci U S A 2019;116:358-66.

68 Masson N, Keeley TP, Giuntoli B et al. Conserved N-terminal cysteine dioxygenases transduce responses to hypoxia in animals and plants. Science 2019;365:65-9.

69 Meadowcroft AM, Cizman B, Holdstock L et al. Daprodustat for anemia: a 24-week, open-label, randomized controlled trial in participants on hemodialysis. Clin Kidney ] 2019:12:139-48.

70 Kaplan J. Roxadustat and anemia of chronic kidney disease. N Engl J Med 2019:381:1070-2.

71 Scheuermann TH, Tomchick DR, Machius M et al. Artificial ligand binding within the HIF2alpha PAS-B domain of the HIF2 transcription factor. Proc Natl Acad Sci U S A 2009;106:450-5.

72 Courtney KD, Infante JR, Lam ET et al. Phase I dose-escalation trial of PT2385, a first-in-class hypoxia-inducible factor-2alpha antagonist in patients with previously treated advanced clear cell renal cell carcinoma. J Clin Oncol 2018;36:867-74.

73 Bishop T, Ratcliffe PJ. HIF hydroxylase pathways in cardiovascular physiology and medicine. Circ Res 2015;117:65-79. 
74 Cheng X, Prange-Barczynska M, Fielding JW et al. Marked and rapid effects of pharmacological HIF-2alpha antagonism on hypoxic ventilatory control. J Clin Investig 2020;130:2237-51.

75 Macias D, Moore S, Crosby A et al. Targeting HIF2alpha-ARNT hetero-dimerisation as a novel therapeutic strategy for pulmonary arterial hypertension. European Respir ] 2021;57: 1902061
76 Haldane JS. The new physiology: Lecture to the Harvey Society of New York. Science 1916;XLIV:619-31.

Address for correspondence: Professor Sir Peter Ratcliffe, Ludwig Institute for Cancer Research, Old Road Campus Research Build, Roosevelt Dr, Headington, Oxford OX3 7DQ, UK.

Email: peter.ratcliffe@ndm.ox.ac.uk 